\title{
LA EXPERIENCIA DEL ADULTO MAYOR DURANTE SU PRIMER AÑO DE INSTITUCIONALIZACIÓN EN UN HOGAR DE CUIDO
}

\author{
Jessica Rivera Arroyo: \\ Zumarie Hernández Bello ${ }^{2}$ \\ Alex Casiano Pagán ${ }^{3}$
}

\section{Resumen}

Este estudio cualitativo de tipo fenomenológico tiene el propósito de auscultar la experiencia de institucionalización y las conductas de adaptación en personas de 65 años o más durante su primer año en un hogar de cuido. Conjuntamente se evaluará el significado que las personas institucionalizadas le adscriben a su experiencia. La muestra esta formada por siete (7) personas de edad avanzada, entre estas seis del género femenino y uno del género masculino, cognitivamente intactos, con un año o menos residiendo en un hogar de cuido de la región sur de Puerto Rico. El estudio discute el proceso de adaptación, sus sentimientos, y el significado atribuido a la experiencia.

[Descriptores: adaptación, envejecimiento, hogar de cuido, institucionalización].

\section{Abstract}

This phenomenological, qualitative study explores the institutionalization experience and adaptive behavior in people 65 years or older during their first year in a nursing home. It will jointly

Puerto Rico.

Trabajadora Social, Escuela Victoria Santiago Pagán, Salinas,

${ }^{2}$ Instructora, Pontificia Universidad Católica de Puerto Rico. Philadelphia.

3 Trabajador Social Clínico, Mercy Life Seminar Health Center, 
assess the meaning that people ascribe to institutionalization experience. The sample consisted of seven (7) older adults, including six females and one male, cognitively intact, with one year or less living in a nursing home in the southern region of Puerto Rico. The study discussed the adaptation process, their feelings, and the meaning they attribute to the experience.

[Keywords: adaptation, aging, nursing home, institutionalization].

\section{Introducción}

Ante el evidente crecimiento de la población de edad avanzada en Puerto Rico, muchas familias han optado por ubicar a sus parientes de edad avanzada en lo que comúnmente se conocen como centros de cuido, égidas $u$ hogares de adultos mayores. Estudios sostienen que para el año 2000 sólo el 2.2 por ciento de las personas de edad avanzada en Puerto Rico se encontraban institucionalizadas (Encuesta de la Comunidad). No obstante, se observó un ascenso en esta cantidad. Entre los años fiscales 2003 al 2006, la cantidad de residentes en establecimientos de cuidado de larga duración aumentó de 11,716 personas institucionalizadas en el año 2004 a 13,006 personas en el año 2005, y a 15,015 en el año 2006, institucionalizados en 693 establecimientos de cuidado de larga duración activos. Dicho total incluye 16 establecimientos geriátricos licenciados por la Administración de Servicios de Salud Mental y contra la Adicción (ASSMCA), 665 instituciones y hogares sustitutos licenciados por el Departamento de la Familia, según establecido en la Ley 94 del 22 de junio de 1977, según enmendada y las 12 Casas de Salud que tienen Certificación Estatal del Departamento de Salud, a través de la Ley 101 y/o Certificación de Medicare.

$\mathrm{La}$ institucionalización, como sostienen Pac, De Vega, Formiga y Mascaro (2006), representa un trastoque a la cotidianidad del adulto mayor que genera niveles de estrés significativos, que, a su vez, requieren la puesta en función de experiencias y estrategias personales para lograr una adecuada adaptación dentro de un hogar de cuido. Según estos autores, los procesos de institucionalización exigen que el adulto mayor se involucre en arduas transiciones y tareas de aceptación y neoadaptación. Al ingresar en un hogar de cuido, dicha población se ve en la necesidad de romper el apego con lo que ha sido su pasado inmediato, a adaptarse a nuevas experiencias de vida, nuevas relaciones interpersonales y aplicar estrategias de afrontamiento para el estrés que le suscita su nuevo entorno. 
Luego de ingresar a un centro geriátrico, el individuo pasa por la disminución en la cantidad de familiares y la falta de recursos (Sepúlveda, Rivas, Bustos, \& Illesca, 2010). Estos investigadores revelan que la internación geriátrica acelera el deterioro, aumenta el grado de dependencia debido a sentimientos de soledad ante el aislamiento de su núcleo familiar, mostrando apatía, y disminución en las actividades sociales, con mayores factores de riesgo como sedentarismo. Por otro lado, el adulto mayor institucionalizado puede presentar incapacidad más perceptible y demanda mayor ayuda para realizar actividades de la vida cotidiana.

La presente investigación tiene el propósito de conocer el significado que los adultos mayores le dan a la experiencia de institucionalización, los temas que emergen de la narrativa de las personas entrevistadas sobre dicha experiencia, y conocer cuáles son las conductas y procesos emocionales de adaptación que asumen los participantes luego de ingresar a un hogar de cuido. El estudio pretende aportar mayor grado de conocimiento a la población puertorriqueña, familiares y profesionales que trabajan con estos individuos de la tercera edad.

Con esta investigación, el Trabajo Social como profesión se beneficiará en la medida en que se pueda comprender y considerar la experiencia de la institucionalización como parte de los protocolos profesionales de intervención con el adulto mayor y su transición hacia otro estilo de vida. De igual forma, intenta proveer mayor conocimiento a los profesionales de la salud, para que estos puedan brindarle a la población bajo estudio la atención necesaria en el proceso de adaptación. Esto a su vez puede fomentar a la creación de programas relacionados a los adultos mayores para que se les pueda guiar en el proceso de ingreso en un centro de cuidado.

\section{Revisión de literatura}

La institucionalización en un hogar de cuido es una experiencia significativa en la vida de una persona. El proceso está lleno de emociones, tanto para la familia como para el adulto mayor. Suárez Campos (2006), explica la respuesta emocional de estas personas ante la necesidad de estar institucionalizados por falta de familiares y/o personas cercanas que los puedan cuidar, resultando en coraje y frustración, al verse impactado su ámbito familiar y los vínculos familiares a los que estaban acostumbrados. En su estudio, los participantes no institucionalizados presentaron mayor nivel de satisfacción que los institucionalizados. La 
autora expone que las relaciones familiares y sociales son más significativas en los individuos no institucionalizados. Esta última es destacada en el deseo del adulto mayor de ser reconocido como ciudadano.

Da Graca da Silva y Roseira Boemer (2009), sostienen que las personas de edad avanzada desean mostrar que ellos no pierden su identidad, no se sienten ancianos, y desean ser reconocidos como ciudadanos. Los participantes de este estudio señalan que tener salud es esencial y que realizan todo lo que está a su alcance para mantener la autonomía, dando importancia al apoyo que reciben de la convivencia y de sus familiares, y valoran su independencia financiera. Estas autoras indican que los individuos de la tercera edad son personas útiles e independientes, las cuales pueden actuar por sí mismas. La pérdida de autonomía podría resultar en complejos trastornos emocionales como la depresión y la ansiedad.

Batista Sáez (2011), señala que los adultos mayores residentes de un hogar de cuido exhibieron una mayor incidencia de síntomas depresivos. Sostiene, además, que no existe diferencia significativa entre las mujeres y los hombres de edad mayor y la incidencia en la sintomatología depresiva. Similarmente, la autonomía funcional se ve afectada debido al aislamiento social. Un estudio de Cader, Guimaraes, Rocha, Vale, Pernambuco y Dantas (2006), plantea que la unión de la persona mayor junto con los hogares de cuido en ocasiones resulta en la disminución de autonomía funcional y la calidad de vida, todo depende de los recursos adaptativos individuales y la actitud con la que se enfrentan a la situación.

De acuerdo con Stefani y Feldberg (2006), los adultos mayores que vivian en sus residencias contaban con mayores recursos adaptativos que los que se encontraban en un hogar de cuido. Los adultos mayores que ingresan a una institución geriátrica probablemente tomen una actitud pasiva como consecuencia de su nuevo entorno y las normas que han de seguir mientras que los no institucionalizados mostraron mayor dificultad en las relaciones interpersonales. En dicho estudio se plantea que la mayoría de la muestra sufrió un evento traumático como una enfermedad grave o la perdida de algún ser querido especialmente la pérdida de su pareja. Álvarez Gómez, Izquierdo Paz, Valdés Mijares, Amurrio Flores y Ortiz Rojas (2008), encontraron en su estudio con residentes en un hogar de cuido 
que, la mayoría de la muestra expresó sentirse no saludables, en relación con el estado de ánimo, autoestima, irritabilidad, sueño e intereses personales. El $77.2 \%$ de la muestra en estudio reportó la autopercepción en la categoría de no saludable, al igual que la percepción de la irritabilidad con un $73.7 \%$. En el aspecto de la autoestima, un $81.1 \%$ indicó no saludable en ese aspecto. Todos estos resultados pueden estar relacionados al afrontamiento de su nuevo entorno.

La socialización es de suma importancia. La institucionalización puede resultar en la pérdida del grupo de apoyo, familia y amistades con las cuales el adulto mayor sostenía una relación significativa. Stoffolani, Orlando y Enria (2006), encontraron en su investigación con mujeres jubiladas que, el $40 \%$ de la muestra mostraba tener alguna relación con alguna institución formal, como centros de jubilados, clubes, entre otros. Un $39 \%$ expuso vivir sola, aunque estuvieran residiendo en un hogar de cuido. Otro resultado de su estudio es que el 79 \% resaltó la importancia de socializar con otros, indicando sobre la necesidad de conversar con amigos, reunirse con otros para cenar, tomar el café, jugar cartas, entre otras cosas. Estos resultados son cónsonos con la aseveración de que las actividades sociales son vitales para una buena salud física y emocional.

Árraga Barrios y Sánchez Villarroel (2007), exponen que a las personas de edad avanzada les gustan las actividades sociales, les entretienen, ocupan su tiempo libre y les permiten aportar a la sociedad y a sus familiares. Los investigadores recalcaron la importancia de permitirle a esta población participar de actividades donde se promueva el dinamismo, promoviendo la satisfacción y la calidad de vida. Peña Pérez, Terán Trillo, Moreno Aguilera y Bazán Castro (2009), exponen que la percepción de calidad de vida está relacionada directamente con las relaciones interpersonales debido a que la persona de edad avanzada que indica ser autosuficiente, mantiene una relación familiar más estrecha y el apoyo de amigos y vecinos, elementos que le proporcionan mayor funcionamiento a pesar de alguna condición de salud.

Cerquera Córdoba (2008), señala que existen elementos correlacionales de deterioro y depresión con eventos significantes del ciclo vital. Plantea que los adultos mayores institucionalizados que no reciben visitas de sus familiares se aíslan de actividades 
propias del asilo. Esto los lleva al retraimiento y la soledad, creándoles sentimientos de abandono y tristeza, que, a su vez, les genera estados de depresión. En el caso de la segunda participante, ésta indicó que su hija la recluyó bajo engaños y no la visita regularmente. Esto es un reflejo de lo expuesto por la autora antes citada y comprueba los sentimientos de coraje e impotencia que sienten estas personas al ser institucionalizadas.

Salarvand, Abedi, Hoseini, Salehi y Karimollani (2008), identificaron tres categorías de experiencias emocionales para las personas de edad avanzada institucionalizadas, basadas en los datos obtenidos en su estudio. Estas son: experiencias emocionales en general, experiencias emocionales al ser ubicados y sentimientos de impotencia. Sobre las experiencias emocionales en la población de estudio, señalaron la importancia de lograr adaptarse a la convivencia dentro de la institución. Por otro lado, indican que el traslado de su residencia a un centro geriátrico era visualizado por los "envejecientes" como un "signo final de fracaso". Finalmente, los autores indicaron que los temores y percepción negativa fue un factor determinante para el proceso de adaptarse a su nuevo entorno.

Rinza González y Manzanares Nieto (2009), establecen que los niveles de independencia/dependencia para realizar actividades diarias son un factor precipitante a la hora de tomar una decisión con respecto al ingreso o no en un hogar de cuido. En su estudio con ancianos institucionalizados encontraron que, quince personas de la muestra estudiada $(40 \%)$, presentaron sintomatología asociada a la depresión, y que luego de recibir tratamiento de un Programa de Rehabilitación Geriátrica, esta disminuyó. Otro hallazgo recopilado de la investigación es que la mayoría de la muestra $(98 \%)$, percibió como normal el apoyo familiar y solo dos participantes $(5 \%)$, percibieron bajo apoyo familiar.

\section{Metodología}

Este estudio cualitativo de tipo fenomenológico utilizó como muestra a siete ( 7 ) personas de edad avanzada, entre estas seis del género femenino y uno del género masculino, con un año o menos residiendo en un hogar de cuido de la región sur de Puerto Rico. Como criterios de inclusión, los participantes debían tener 65 años o más, estar institucionalizado en un centro geriátrico, no estar recibiendo servicios de salud mental y poseer capacidad verbal narrativa. La selección de la muestra se realizó 
contando con la disposición de los participantes. Para acceder a la misma, se contactó a los directores de los hogares donde se llevó a cabo dicho estudio ubicados en los Municipios de Arroyo y Guayama.

En esta investigación se utilizaron tres instrumentos para recopilar la información. El primero fue una hoja de consentimiento donde la persona autorizó la entrevista y se le explicó el propósito, duración y alcance de la misma (ver Apéndice A Hoja de Consentimiento). El segundo instrumento se constituyó por una hoja de datos sociodemográficos donde se recopiló la información personal, género, edad, estado civil, tiempo de institucionalización, entre otros (ver Apéndice B Hoja de datos sociodemográficos). El tercer instrumento estuvo formado por una guía de 14 preguntas abiertas que sirvieron de guía para las entrevistas semiestruscturadas llevadas a cabo (ver Apéndice C Guión de Preguntas).

La información brindada por los participantes se transcribió de forma ad verbatim. Esto con el propósito de analizar los datos recopilados. Asimismo, se observaron las similitudes y las diferencias de las respuestas, las cuales fueron sostenidas con la revisión de literatura. Como técnica de análisis, se utilizó el análisis de contenido. Según Kerlinger y Lee (2006), el análisis de contenido ayuda a la codificación y a la preparación de datos recopilados de forma verbal. Estos autores señalan que mediante las preguntas abiertas se pueden categorizar los resultados obtenidos. Los subtemas emergentes fueron: significado, impacto, apoyo y adaptación.

\section{Resultados}

\section{El significado de la de institucionalización según los participantes}

La primera categoría emergente de las entrevistas realizadas tuvo como propósito indagar y comprender el significado de la experiencia de institucionalización durante su primer año en un hogar de cuido, según la primera pregunta de investigación. Los resultados del estudio demuestran que para todos los participantes la experiencia de institucionalización durante su primer año en un hogar de cuido significó una experiencia emocionalmente devastadora, donde el estar lejos de sus seres queridos y entorno familiar, les ocasionó tristeza, amargura y melancolía, aunque tengan que finalmente aceptar 
su destino. En el caso de la segunda participante, también le ocasionó sentimientos despectivos hacia su hija, manifestando coraje por esta haberla recluido en la institución.

Impacto de los participantes al momento de ser institucionalizados por primera vez

La segunda categoría obtenida de los resultados de las entrevistas en el presente estudio tuvo como objetivo describir el impacto a los participantes de edad avanzada, al momento de ser institucionalizados por primera vez. El impacto manifestado en los siete participantes no fue precisamente uno positivo. Los hallazgos revelaron que en todos los participantes resultó ser un impacto chocante, específicamente en el ámbito familiar, aunque no inesperado. Esto porque todos entendían que sus seres queridos no podían atenderlos debido a sus responsabilidades y obligaciones cotidianas.

Sin embargo, aun al momento, todos continúan añorando estar en sus casas y/o con sus seres queridos, lo que demuestra el fuerte impacto recibido al prácticamente, quedar solos y al cuidado de extraños, aunque reciban visitas de los familiares. Al ser formulada esta pregunta, de describir los cambios que han experimentado como resultado de pertenecer a la institución donde residen, la primera, tercera y el quinto participante coincidieron en que no hay nada como su propio hogar, entre otras cosas. En la entrevista con la segunda participante, los hallazgos reflejaron el impacto devastador de ser institucionalizada, al sentirse engañada y despreciada por su hija, algo que para ella resultó emocionalmente impactante, expresando en un momento dado "Mi hija por no limpiarme ella me metió al hogar y eso yo lo siento...".

También los hallazgos indicaron que la segunda y la séptima participante asociaron y experimentaron en alguna medida, un impacto y deterioro en su estado de salud al estar institucionalizadas. La séptima participante expresó haber sentido una disminución en su peso corporal y sentirse "más patrá". Mientras que, la segunda participante indicó que en estos momentos se pasa en una silla de ruedas todo el día sin poder caminar, mientras que antes cuando residía en su propio hogar caminaba y realizaba sus tareas.

También, el estudio encontró que, se impactó la vida cotidiana y el ámbito social de los participantes, al no poder participar de algunas actividades y salidas que llevaban a cabo. 
Esto se aprecia, por ejemplo, en las respuestas manifestadas por la tercera y séptima participante, donde indican que antes de ingresar al hogar de cuido podian asistir a la iglesia, pero al momento de las entrevistas, aún se les hacía imposible asistir. Por otro lado, se afectó la alimentación de los participantes, como muy bien expresó la primera participante, al rememorar cómo le satisfacía y estaba acostumbrada a ciertos alimentos, teniendo ahora que consumir lo que les ofrecen dentro de la institución, aunque no le agrade. En el caso de la segunda participante, se vio impactada su percepción de lo que era la jubilación, expresó que veía la jubilación de otra forma como, volver a asumir las riendas de su propio hogar, y no tener que jubilarse en un hogar de cuido. Para el único participante masculino, el quinto, el impacto ha sido tan devastador que, expresa sentirse como si le hubieran arrebatado su vida, encerrado y sin libertad, en adición abandonado por su pareja, con la que convivía antes de entrar al hogar.

\section{Apoyo de los participantes durante la instituciona- lización}

La tercera categoría que se desprende de las entrevistas y fue analizada para fines investigativos, tuvo como propósito describir y comprender el tipo y/o red de apoyo con que cuentan los participantes durante su institucionalización. La investigación arrojó que seis de los participantes mantienen una buena relación con sus familiares, los que constituyen su principal red de apoyo, contrario a la segunda participante, que indicó no tener una relación positiva con algunos de sus familiares, en especial, con su hija.

Según los hallazgos encontrados, todos los participantes sienten el apoyo del personal del hogar al cual pertenecen, especialmente, el respaldo y atención de las propietarias. Los resultados también revelaron que los participantes reciben visitas de familiares y que durante estas visitas se sienten contentos y emocionados, como lo expresó la primera participante al responder "pues los familiares". La segunda participante contestó, "Pues mi hija, fulana... y mi hijo fulano". La tercera participante respondió de la siguiente manera, "El miércoles me vinieron a visitar mi cuñado con la esposa... me visitan personas que son conocidos aquí...". Mientras que la cuarta participante respondió, "Mis hijos... siento alegría y tranquilidad porque sé que ellos vienen a visitarme...". El quinto participante señaló que su hijo 
lo visita junto a su nuera y expresó que se siente "emocionado" durante las visitas. La sexta participante recalcó, "Cuando me visitan, me pongo contenta". Mientras que la séptima participante refirió sentirse, "Alegre, me gusta estar con mi familia cuando fulana me viene a buscar algunos sábados".

\section{Proceso de adaptación durante su primer año en el hogar de cuido.}

La cuarta y última categoría desprendida de las entrevistas realizadas tuvo como fin describir el proceso de adaptación que experimentaron los participantes durante el primer año residiendo en el hogar de cuido. Se encontró similitud en seis de los participantes, al estos responder que no han realizado cambio alguno para adaptarse a su nuevo entorno. En la primera, se encontró que la misma señala tener fe y esperanzas de regresar a su propio hogar. Mientras que, cinco participantes prefieren responder dejar todo como está. En la segunda participante, el estudio reflejó que sí ha realizado cambios para poder adaptarse a su nuevo entorno, cambiándose de habitación y escogiendo una con mayor privacidad.

Ante la pregunta sobre, ¿Qué cosas le dan mayor satisfacción en esta etapa de vida? Teniendo en cuenta, que dependiendo de qué cosas le causan satisfacción, pueden ayudar a los participantes en el proceso de adaptación dentro del hogar de cuido. La investigación arrojó que cinco de los participantes respondieron que el poder ver a sus hijos, es la mayor satisfacción en estos momentos de sus vidas. Estos hallazgos se pueden comprobar según las respuestas de los participantes como por ejemplo, la primera, tercera y séptima participante indicaron " $m i s$ hijas". La cuarta participante expresó, "cuando ellos vienen a verme". El quinto participante respondió "emocionado porque me vinieron a visitar". Por otro lado la segunda participante respondió "ninguna", y la sexta participante expresó que en estos momentos de su vida lo que mayor satisfacción le dá es el poder sentirse acompañada, "no estoy sola, cualquier cosa esa gente están pendiente...".

Sobre los aspectos que les provocan insatisfacción a los participantes en esta etapa de vida, la primera, segunda, tercera, cuarta y quinto participante, respondieron sentir insatisfacción al tener que residir en un hogar de cuido durante la presente etapa de su vida. La primera y segunda participante, expresaron sentir insatisfacción al estar en la institución de cuido. La tercera 
participante respondió, "yo no quería salir de mi casa...". En la cuarta participante el estudio encontró que ella desearía salir del hogar de cuido, pero solo si uno de sus hijos se lo propone. Los hallazgos revelaron que el quinto participante señaló insatisfacción por estar dentro del hogar de cuido sin ayuda de sus familiares. En la sexta y séptima participante el estudio reflejó que al momento no sentían insatisfacción en esta etapa de su vida.

Al formular la pregunta ¿Cambiarias algo de su vida? ¿Por qué?, el estudio encontró que la segunda, tercera y cuarta participante desearian regresar a su propio hogar, porque nunca pensaron estar fuera de ellos en esta etapa de su vida. Los hallazgos encontraron que el quinto y la sexta participante expresaron que no cambiarían algo en su vida. Otro hallazgo encontrado en el estudio es que la primera participante respondió que si pudiera cambiaría toda su vida. La séptima participante no tuvo comentarios a la pregunta formulada.

\section{Discusión}

En el resultado final, se encontró que para todos los participantes, el significado de la experiencia de institucionalización durante su primer año en un hogar de cuido, fue una experiencia emocionalmente devastadora, donde el estar lejos de sus seres queridos y entorno familiar, les ocasionó tristeza, amargura y melancolía, según expresaron durante las entrevistas, aunque tengan que finalmente aceptar su destino. En el caso de la segunda participante, también le ocasionó sentimientos despectivos hacia su hija, manifestando coraje por esta haberla recluido en la institución.

Entre los hallazgos del estudio, se pudo apreciar como la segunda y la séptima participante asociaron y experimentaron en alguna medida, un impacto y deterioro en su estado de salud al estar institucionalizadas. La séptima participante expresó haber sentido una disminución en su peso corporal y sentirse "más patrá". Mientras que la segunda participante indicó que en estos momentos se pasa en una silla de ruedas todo el día sin poder caminar, mientras que antes, cuando residía en su propio hogar, caminaba y realizaba sus tareas. Similarmente, al verse limitados y no poder actuar libremente, tanto las participantes féminas como el único participante masculino, presentaron en algún momento dado tendencias a deprimirse. Esto especialmente manifestado en la cuarta participante, al punto de expresar que sería mejor "si un carro me pisara", y en la segunda participante al expresar 
prácticamente sentirse invisible, ya prácticamente no habla con nadie y expresó sentirse "como muda". En el participante masculino se pudo apreciar su tristeza al este expresar, "Más mal, no es lo mismo estar libre que estar aquí encerrao, donde tú puedes ir donde tú quieras y aquí tú no puedes hacerlo".

El estudio revela que los participantes no habían realizado cambio alguno para adaptarse a su nuevo entorno y que sencillamente dejaban pasar el tiempo. La primera participante expresó tener fe y esperanzas de regresar a su propio hogar. En la segunda participante, el estudio encontró que sí ha realizado cambios para poder adaptarse a su nuevo entorno, como cambiar de habitación y escoger una con mayor privacidad. Añadió que, fuera de eso, no ha llevado a cabo ningún cambio significativo con el fin de adaptarse a su nuevo entorno, al contrario. Esta indicó sentir rabia y coraje por tener que estar recluida en un hogar de cuido, actitud que proyecta dificultad en su proceso de adaptación a su nueva realidad.

Según los hallazgos de nuestra investigación, la institucionalización para un anciano/a puede significar un evento traumático y devastador al tener que salir de su propia residencia sintiéndose abandonado por sus seres queridos. Este último aspecto es evidente aunque los participantes no quisieron abiertamente expresarlo, justificando, en la mayoría de los casos, el que sus hijos tienen que vivir su vida, trabajar y/o atender sus respectivas familias. Irónicamente, todos los y las participantes del estudio tienen la esperanza de algún día vivir con sus seres queridos, si estos deciden llevárselos a residir con ellos. Esto quedó planteado dramáticamente en la segunda participante, siendo la más afectada de todos.

\section{Recomendaciones para la práctica del Trabajo Social}

La siguiente investigación presenta algunas recomendaciones útiles para la práctica del Trabajo Social en Puerto Rico. Es necesario validar la relevancia del presente estudio y llevar a cabo más investigaciones cualitativas en el campo del trabajo social con adultos mayores en Puerto Rico. De igual forma, es necesario promover política pública para las personas de edad avanzada en el proceso de adaptación durante el primer año al ser institucionalizados. Como trabajadores sociales tenemos una responsabilidad con la comunidad en general, es imperativo promover talleres educativos dirigidos a los familiares y cuidadores principales con el objetivo de prevenir, en alguna 
medida, el que la experiencia de institucionalización sea una traumática y emocionalmente devastadora para las personas de edad avanzada.

\section{Conclusión}

Los hallazgos revelaron que con respecto al significado de la experiencia de institucionalización y conducta de adaptación en personas de 65 años o más durante su primer año en un hogar de cuido, hubo similitud en los siete participantes. La similitud consistió en su compartida percepción de que la institucionalización fue emocionalmente devastadora y traumática, al tener que enfrentar un nuevo ámbito social. Por otro lado, se pudo evidenciar que los sentimientos de tristeza, amargura y melancolía forman parte del significado de la experiencia de institucionalización, sentimientos que eran atenuados con la visita de familiares.

De igual forma, se encontró que para los y las participantes del estudio, este nuevo entorno ha cambiado la manera de ver la vida, pero no necesariamente de forma positiva. Esto no solo por sus experiencias de vida anteriores y en el ciclo de la vida en que se encuentran al momento, donde el tener una edad avanzada les provoca cansancio y dolencias físicas, entre otras cosas, sino que ahora, además,, se sienten parcialmente abandonados, traicionados (como lo es el caso de la segunda participante), y hasta cierta forma defraudados con la realidad que les ha tocado vivir durante esta etapa tan vulnerable y delicada de la vida. Etapa donde debieran ser atendidos y recompensados con el mismo amor y alegría que sienten y les brindan ellos, cuando hijos y familiares vienen a visitarlos. 


\section{Referencias}

Álvarez Gómez, Tamara, Izquierdo Paz, Jorge Enrique, Valdés Mijares, Marisel de la C., Amurrio Flores, Ricardo E.\& Ortiz Rojas, Edwin Giovany. (2008). Resultados del estudio sobre la autopercepción y validismo en ancianos residentes en el Hogar Provincia Ever Riverol Bernal. Gaceta Medica Espirituana. 10 (3), 1-5.

Árraga Barrios, Marisela Virginia, \& Sánchez Villarroel, Marhilde. (2007). Recreación y calidad de vida en adultos mayores que viven en instituciones geriátricas y en sus hogares. Un estudio comparativo. Espacio Abierto. 16, 737-756.

Batista Sáez, Yomaira. (2011). Relación entre el Lugar de Vivienda y La Depresión en una Muestra de Personas Mayores de 65 años del Área Sur de Puerto Rico. PUCPR Proyecto de Investigación sometido al Colegio de Estudios Graduados en Ciencias de la Conducta y Asuntos de la Comunidad de la Pontificia Universidad Católica de Puerto Rico para obstar por el grado de Maestría en Trabajo Social Clínico Mayo 2011.

Cader, Samaria Ali, Guimaraes, Andrea Carmen, Rocha, Cristiano, Vale, Rodrigo, Pernambuco, Carlos, \& Dantas, Estelio Henrique. (2006). Perfil de la calidad de la vida y autonomía funcional de los ancianos asilados en una institución filantrópica en el municipio de Rio de Janeiro. Fitness Performance. 4, 256-261.

Cerquera Córdoba, Ana Mercedes. (2008). Relación entre los procesos cognitivos y el nivel de depresión en las adultas mayores institucionalizadas en el Asilo San Antonio de Burcaramanga. Universitas Psicológica. 7 (1), 1-8.

Da Graca da Silva, María, \& Roseira Boemer, Magali (2009). The experience of aging: a Phenomenological perspective. Mendeley.17 (3), 380-386.

Encuesta de la Comunidad (2006) Encuesta sobre la Comunidad de Puerto Rico del 2005-2009 -Estimados de 5 Años, Estimados Demográficosy de Vivienda de laACS:2005-2009. Recuperadoel 15 de septiembre de 2011 de http://factfinder.census.gov/servlet/ ADPTable?_bm=n\&_lang=es\&qr_name=ACS2009_5YR G00_DP5YR5\&ds_name=ACS_2009_5YR_G00_\&geo_ id $=04000$ US72

Kerlinger Fred N., \& Lee Howard B. (2006). Investigación del Comportamiento: Métodos de Investigación en Ciencias Sociales. México, D.F. McGraw Hill/Interamericana.

Pac, Pilar, de Vega, Pilar, Formiga, Frances, \& Mascaro, Jordi. (2006). Motivos de ingreso en residencias geriátricas en Calahorra (La Rioja). Revista Multidisciplinaria de Gerontología. 16 (1), 32-36.

Peña Pérez, Belem, Terán Trillo, Margarita, Moreno Aguilera Fernando, \& Bazán Castro, Manuel. (2009). Auto percepción de la calidad de vida del adulto mayor en la Clínica de Medicina Familiar Oriente 
del ISSSTE. Revista de Especialidades Médico-Quirúrgicas. 14 (2), 53-61.

Rinza González, Mercedes, \& Manzanares Nieto, Diana. (2009). Programa integral de rehabilitación para la mejora de la calidad de vida en pacientes geriátricos. Revista Mexicana de Medicina Física y Rehabilitación. 21 (1) 21-22.

Salarvand, Shahin, Abedi, Heidar, Hoseine, Habibollahi, Salehi, Shayesteh, \& Karimollahi, Mansooreh. (2008). The emotional experiences of elderly people living in nursing homes. Annals of General Psychiatry.7 (1), 1.

Sepúlveda, Catalina, Rivas, Edith, Bustos, Luis, \& Illesca, Monica. (2010). Perfil socio familiar en adultos mayores institucionalizados. Temuco, padre las casas y nueva imperial. Ciencia y Enfermería. 3, 49-61.

Stefani, Dorina, \& Feldberg, Carolina. (2006). Estrés y estilo de afrontamiento en la vejez: un estudiocomparativo en senescentes argentinos institucionalizados y no institucionalizados. Anales de Psicología. 22 (2), 267-272.

Stoffolani, Claudio, Orlando, Marta, \& Enria, Graciela. (2006). Adultos mayores. Situación actual y perspectivas futuras. RevMultGerontol. 6-11.

Suarez Campo, Vanessa. (2006). Nivel de Satisfacción de Vida de Personas de Edad Avanzada Institucionalizados y no Institucionalizados. Tesis Sometida al Instituto de Estudios Graduados en Ciencias de la Conducta y Asuntos de la comunidad de la Pontificia Universidad Católica de Puerto Rico para optar por el grado de Maestría en Trabajo Social Clínico Mayo 2006. 
Apéndice A

PONTIFICIA UNIVERSIDAD CATÓLICA DE PUERTO RICO JUNTA DE REVISIÓN INSTITUCIONAL

2250 Ave. Las Américas, Suite 571

Ponce, PR 00717-9997

Teléfono 787-841-2000 Ext. 1210

Fax (787) 651-4029

Correo Electrónico: JRInst।@email.pucpr.edu

\section{CONSENTIMIENTO PARA REALIZAR LA INVESTIGACIÓN}

Certifico que he leído el procedimiento para llevar a cabo la investigación titulada: El Significado de la Experiencia de Institucionalización y Conductas de Adaptación en Personas de 65 Años o más Durante su Primer año en un Hogar de Cuido de Jessica Rivera Arroyo. La investigadora me ha explicado el propósito de su investigación y ha contestado mis preguntas relacionadas a la misma. Estoy de acuerdo en participar de la investigación sobre la experiencia y el proceso de adaptación en el hogar de cuido. Además, he recibido copia de este procedimiento.

Participante

Fecha 


\section{Apéndice B}

\section{Instrucciones}

La siguiente investigación recopilará información, por medio de una hoja de datos socios demográficos. El entrevistador le preguntará al participante el nombre, edad, fecha de nacimiento, estado civil, religión a la cual pertenece y si tiene hijos dentro o fuera de Puerto Rico. Esto con el propósito de poder categorizar los datos obtenidos. Luego se procederá a una entrevista, la cual estará representada por 13 preguntas abiertas. Tendrá una duración de aproximadamente 45 minutos. Toda la información brindada será voluntariamente y guardada de forma confidencial. El participante tendrá el derecho de contestar lo que entienda correspondiente y a negarse a continuar con la entrevista, no será penalizado. Este estudio no llevará riesgos, ni se ofrecerá beneficios o remuneración alguna. Los dados recopilados serán compartidos y los mismos estarán disponibles en la Sala de Puerto Rico en la Biblioteca Encarnación Valdés de la Pontificia Universidad Católica de Puerto Rico.

Hoja de Datos Socio Demográficos

Nombre

Edad

Estado Civil

Religión

Hijos 


\section{Apéndice $\mathbf{C}$}

\section{Guía de Preguntas}

Instrucciones:

El instrumento utilizado será una guía de catorce (14) preguntas abiertas, las cuales serán leídas por la entrevistadora. Los participantes contestarán según sus conocimientos y su interés. Tiene derecho a no querer contestar algunas de las preguntas. Toda la información brindada será voluntariamente y guardada de forma confidencial. La duración de la entrevista será de 45 minutos aproximadamente. Estas preguntas tienen el propósito de investigar cómo fue que el participante ingreso al hogar de cuido, como era su vida entes de pertenecer al centro, cuales ha sido los cambios experimentados, como esto ha cambiado su vida, si la persona tiene hijos, como es su relación familiar y con sus compañeros dentro del hogar, cual es el apoyo recibido, si recibe alguna clase de visita, cual ha sido las experiencias que le han dado mayor satisfacción en la vida, cuáles son los aspectos que le provocan insatisfacción en esta etapa de vida y si cambiaria algo de su vida. Todas estas preguntas ayudaran a categorizar los datos obtenidos y poder brindar una información relacionada a la población de estudio.

1. Describa como era su vida antes de ingresar a éste hogar.

2. Describa cómo fue que llegó al centro y por qué.

3. Describa qué cambios ha sentido luego de pertenecer a la institución.

4. Describa cómo este nuevo entorno ha cambiado o no la manera de ver la vida.

5. Describa como era su relación con sus hijos antes de ingresar al hogar

6. Describa cómo es su relación actual con sus familiares.

7. Describa cómo es su relación con sus compañeros/as dentro del hogar.

8. Describa que cambios y ajustes ha realizado para sentirse mejor en este hogar

9. En términos generales, describa cómo se siente

10. Describa qué clase de apoyo tienes en estos momentos

11. ¿Quiénes lo visitan? Describa cómo se siente durante estas visitas.

12. ¿Qué cosas le dan mayor satisfacción en esta etapa de vida?

13. ¿Cuáles son los aspectos que le provocan insatisfacción en esta etapa de vida?

14. ¿Cambiaría algo de su vida? ¿Por qué? 